\title{
Object-Based Land Cover Classification Using Airborne Lidar and Different Spectral Images
}

\author{
Tee-Ann Teo* and Chun-Hsuan Huang \\ Department of Civil Engineering, National Chiao Tung University, Hsinchu, Taiwan, R.O.C.
}

Received 18 August 2015, revised 7 January 2016, accepted 29 January 2016

\begin{abstract}
Both land cover spectral information and 3D surface information can be obtained efficiently via remote sensing technologies. Spectral images provide spectral features whereas lidar point clouds contain 3D spatial features. Therefore, the multisensor data can be integrated to obtain useful information for different applications. This study integrates lidar with different spectral features for land cover classification. Because different spectral images have different characteristics, this study used hyperspectral images, 4- and 8-band WorldView-2 multispectral images, to distinguish different land covers. The major works include features selection, object-based classification, and evaluation. In features selection appropriate features were selected according to the land cover characteristics. Object-based classification was implemented using image segmentation and supervised classification. Finally, different combinations were evaluated using reference data to provide comprehensive analyses. We use ITRES CASI-1500 airborne hyperspectral images, WorldView-2 multispectral images and Optech ALTM Pegasus in this study. The experiment compared the results with and without data fusion. The importance of different spectral features is also discussed. In summary, different land covers with similar spectral features can be identified using lidar spatial features. Spectral image integration with lidar data may improve land cover classification accuracy.
\end{abstract}

Key words: Lidar, Hyperspectral image, WorldView-2, Object-based classification

Citation: Teo, T. A. and C. H. Huang, 2016: Object-based land cover classification using airborne lidar and different spectral images. Terr. Atmos. Ocean. Sci., 27, 491-504, doi: 10.3319/TAO.2016.01.29.01(ISRS)

\section{INTRODUCTION}

Land cover maps are important infrastructure features in environmental studies. Because land cover changes rapidly, reliable land cover maps must be developed effectively and precisely. Remote sensing technologies can efficiently obtain the spectral and shape properties of ground objects. Because this information is suitable for large area land cover classification, remote sensing technologies can be used to investigate circumstances and changes in land surface for land management.

Spectral image and lidar point cloud data are commonly used in land cover classification. For spectral images different land covers can be separated using an image classification technique that analyzes the spectral information to obtain the land cover. The spectral resolution significantly affects the results. For lidar point clouds different land covers can be distinguished by shape and intensity information. Different

\footnotetext{
* Corresponding author

E-mail:tateo@mail.nctu.edu.tw
}

land covers can be classified by simultaneously considering both spectral and shape information. Therefore, lidar point clouds and its complementary counterpart, spectral images, are usually integrated in land cover classification (Chen et al. 2009, 2012; Debes et al. 2014; Liao et al. 2015).

The main focus in optical sensor development is to improve spatial and spectral resolutions by developing hyperspectral sensors with high spatial resolution. Improved spatial and spectral resolutions are beneficial for interpreting types of land cover and are used to improve the potential of image interpretation and classification. Because satellite images cover larger area and are usually selected for land cover classification, most of the very high resolution satellites (e.g., IKONOS, QuickBird, OrbView-3) acquire 4 spectral bands (blue, green, red, and near infra-red channels) to compose a multispectral image. Some of the high resolution satellites, such as WorldView-2, can acquire more than 8 spectral bands (coastal, blue, green, yellow, red, red edge, nir1, and nir2) to improve the image interpretation capability . 
Landsat- 8 also increases the multispectral and thermal images from 7 to 10 different bands. Several studies have indicated that the 8 bands of multispectral WorldView-2 images can improve classification accuracy in urban areas (Longbotham et al. 2012) as well as forested areas (Immitzer et al. 2012). Hyperspectral imagery additionally provides more detailed spectral information than discrete multispectral imagery and has high potential to identify vegetation species using spectral information (Clark et al. 2005; Govender et al. 2008). The challenge of using multispectral images for image classification is the extraction of useful features (also called spectral signatures) from limited discrete spectral bands. By contrast, the challenges of using hyperspectral images for image classification are related to the high dimensionality of the hyperspectral data and the limited availability of training samples (Bioucas-Dias et al. 2013). Both multispectral and hyperspectral images are limited in their representation of surface information rather than internal properties.

Different techniques have been developed to fuse lidar and imagery in land cover classification (Gamba 2014). This fusion can be classified into three categories based on strategy: pixel data fusion, feature level fusion, and multiapproach fusion (Pohl and Van Genderen 1998). Pixel data fusion is a low level data fusion that directly combines two datasets [i.e., lidar digital surface modeling (DSM) and orthoimage] via world coordinates; feature level fusion integrates image-derived features (e.g., Normalized Difference Vegetation Index, NDVI) and lidar-derived features (e.g. penetrating rate) to gain more useful information; and multi-approach fusion combines different processing methods (e.g., unsupervised and supervised classifications) for precise classification.

Data fusion for lidar and imagery can be classified into two categories based on datasets: fusion of lidar and multispectral images (Bork and Su 2007), lidar and hyperspectra (Dalponte et al. 2008). Lidar technology rapidly obtains high-density $3 \mathrm{D}$ point clouds and efficiently provides 3D surface information that can assist the identification of land cover (Guo et al. 2011) because every ground object has a characteristic height. The integration of lidar and spectral information is therefore beneficial in land cover classification. Rahman et al. (2013) compared the results of support vector machine (SVM) and maximum likelihood (ML) classifiers for 7 classes using airborne lidar and red-green-blue (RGB) aerial images. SVM produced better classification results than the ML method. In addition, the combination of lidar-derived features and multispectral images produced better results than classification based on single data. Liao et al. (2014) combined features of lidar and hyperspectral images using a graph-based feature fusion approach. Experimental results demonstrated the effectiveness of multisensor data classification.

Image classification development includes pixel-based classification that performs image analyses pixel-by-pixel and object-based classification that combines similar pixels into objects through image segmentation. The advantages of object-based classification have been proven from previous studies (Myint et al. 2011; Duro et al. 2012). Through image segmentation the related image pixels are combined into image objects that provide the objects' attributes and shape to identify different land covers (Ke et al. 2010). Object-based classification is therefore more flexible than traditional pixel-based classification (Blaschke 2010).

Several studies have indicated that lidar and spectral image fusion may improve land cover classification accuracy (Bork and Su 2007; Geerling et al. 2007; Zhou 2013). However, most studies focused on multispectral or hyperspectral images and comparisons between lidar with different spectral images are sparse. This study uses object-based classification that considers both spatial and spectral features to distinguish different land covers. The scope of this study includes: (1) compare the accuracies of classification for lidar fusion with different spectral images (i.e., traditional 4-band multispectral, advanced 8-band multispectral, and hyperspectral images); and (2) analyze the importance of different lidar and spectral features in object-based classification.

This research compares the results from object-based classification by integrating lidar with different spectral images including 4-band multispectral WorldView-2 images, 8-band multispectral WorldView-2 images, and 72-band ITRES CASI1500 hyperspectral images. This study adopted the feature-level fusion approach. The proposed scheme includes feature selection, image segmentation and image classification. Lidar data and spectral images were first coregistered in the same coordinate system. Twelve different land type covers were then predefined as target classes for classification. After the iterative segmentation we used the extracted objects to calculate the different features for classification. A supervised nearest neighbor (NN) method with different features was adopted to classify different land covers. The confusion matrix was generated by ground truth information and verified by comparing lidar and different spectral image classifications.

\section{STUDY AREA AND DATASET}

The test area (Fig. 1) is located at the junction of Chiayi and Kaohsiung Counties in Taiwan, a mountainous area near Tseng-Wen Watershed with different types of vegetation, rivers, villages, and developed farmland. The test data includes lidar, multispectral and hyperspectral images (Table 1). The spatial resolutions of lidar, hyperspectral, and WorldView-2 multispectral images are 1, 1, and $2 \mathrm{~m}$, respectively. The size of the targets for classification was larger than $2 \mathrm{~m}$ so that all of the targets could be identified using a spectral image with $2 \mathrm{~m}$ spatial resolution. Gao and Mas (2008) indicated that the impact of similar resolutions is limited in object-based classification. To minimize the 
impact of different resolutions this study used object-based image classification to merge the individual pixels into regions for classification. All of the comparisons from different spectral images are based on object-based classification.

\subsection{Lidar}

Lidar data were collected by Optech ALTM Pegasus. Lidar was interpolated onto a $1 \mathrm{~m}$ raster grid to integrate lidar and spectral images. The lidar features include normalized DSM (nDSM), roughness, intensity, echo ratio and other features. In lidar data processing point clouds are classified as ground points and non-ground points for digital terrain modeling (DTM) and DSM. DSM can be used for image ortho-rectification. The nDSM can be calculated by subtracting DSM from DTM.

\subsection{Multispectral Image}

Multispectral images were collected by the WorldView-2 satellite, consisting of 8 bands of discrete spectral data from 400 - $1050 \mathrm{~nm}$ with $2 \mathrm{~m}$ spatial resolution. The spectral bands include coastal, blue, green, yellow, red, red edge, nir1, and nir2. We selected blue, green, red, and nir1 to represent the traditional 4-band images to compare the results from 8 and 4 bands. The multispectral images pre-processing includes radiometric and geometric corrections. The radiometric correction converted the gray value to reflectance (Updike and Comp 2010) using metadata of WorldView-2 image. The geometric correction was performed using the rational function model (RFM) (Chen et al. 2007) with ground control points and lidar DSM.

\subsection{Hyperspectral Image}

Hyperspectral images in this study were collected by ITRES CASI 1500 with 72 image bands and a spectral wavelength range from 362.8 - $1051.3 \mathrm{~nm}$. The hyper-spectral image pre-processing included radiometric and geometric corrections. The ATCOR4 method was applied for radiation correction to obtain the same ground surface reflectivity from every strip. This method considers a variety of factors including incident angle, terrain effects, and atmospheric effects, resulting in an overall decrease in effects from these sources (Hong et al. 2013). Furthermore, hyperspectral images have a large number of bands; therefore, to avoid dimensionality in the limited training area, the minimum noise fraction transformation (MNF) method was used to reduce the number of hyper-spectral image dimensions. The first 7 MNF bands were selected for classification. (a)

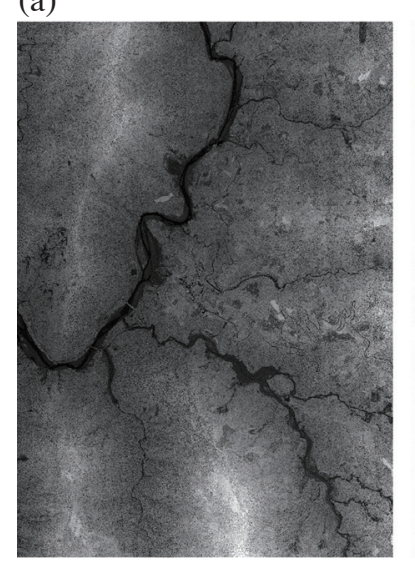

(b)

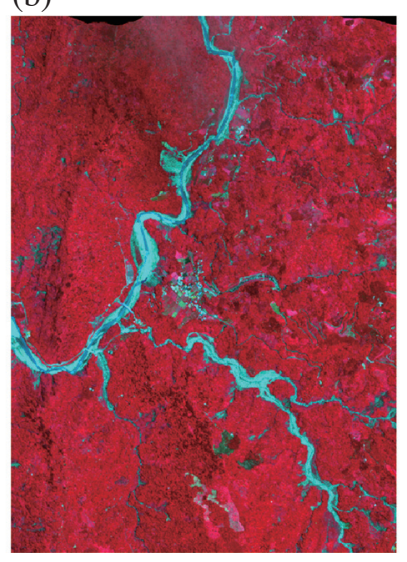

(c)

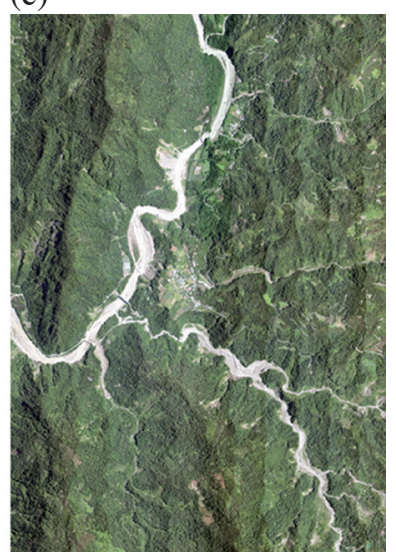

Fig. 1. Test data (a) Lidar intensity, (b) WorldView-2 (G, R, NIR1), (c) CASI (B, G, R). (Color online only)

Table 1. Summary of test data.

\begin{tabular}{c|c|c|c}
\hline & Lidar & Multispectral image & Hyperspectral image \\
\hline Sensor & Optech ALTM Pegasus & WorldView-2 & ITRES CASI 1500 \\
Date & $23-24$ July 2013 & 4 August 2013 & 24 July 2013 \\
Spectral information & $1064 \mathrm{~nm}$ & $400-1050 \mathrm{~nm}$ & $362.8-1051.3 \mathrm{~nm}$ \\
Number of band & 1 & 8 & 72 \\
Spatial information & $1 \mathrm{~m}$ grid & 2 m orthoimage & $1 \mathrm{~m}$ orthoimage \\
Features & DTM, DSM, nDSM, Roughness, Echo ratio, Intensity, Texture & Spectral band, NDVI, Texture & MNF, NDVI, Texture \\
\hline
\end{tabular}




\subsection{Targets of Classification}

According to the test area characteristics and recognizable features, the study area was divided into vegetation including forest, grass and various types of crops, and non-vegetation areas including bare ground, rivers and various types of man-made structures (Fig. 2). We identified 12 land cover types and selected several training areas for each. The mean spectral responses (Fig. 3) were produced based on whole training datasets. Different colors indicate different land covers. The 8-band and hyper-spectral image trends are similar due to the same wavelength coverage $(400-1050 \mathrm{~nm})$, but the hyper-spectral image spectral responses are more detailed than 8-band images. The 8-band image separability is better than that of traditional 4-band images, a clear advantage. For 4-band multispectral images the separability among vegetation types (e.g., areca and broadleaf) is low. The mean 8-band multispectral image spectral responses are better than those for 4-band images. The separabilities at the red-edge, NIR1 and NIR2 are superior to that of other bands. Overall, the hyper-spectral images show better spectral responses in discriminating different land covers. The training and evaluation data (Fig. 4) were carefully selected by field work and $0.5 \mathrm{~m}$ aerial orthoimaging. For the areas that could be assessed we determined 53 check regions using field work. We also used $0.5 \mathrm{~m}$ aerial orthoimages to identify the well-defined targets as check regions to have well-distributed check regions. We summarized the areas used for training and verification of each class (Table 2).

(a)

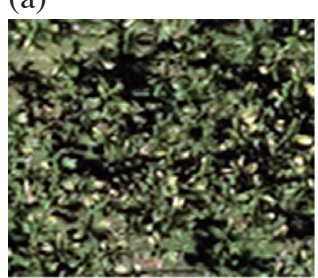

(e)

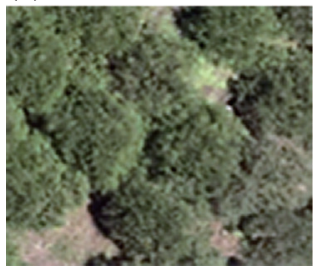

(i)

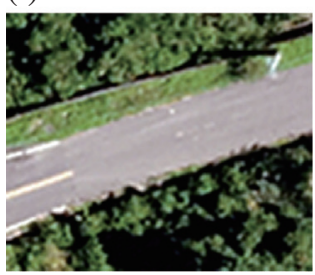

(b)

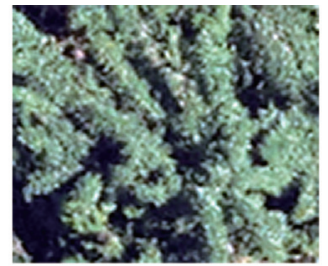

(f)

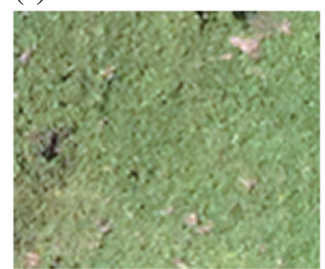

(j)

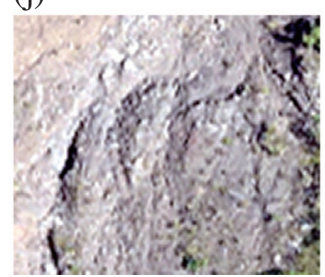

\section{PROPOSED SCHEME}

The framework (Fig. 5) consists of three major parts: (1) data combination; (2) object-based classification; and (3) evaluation. We combined lidar with different spectral images to understand the performance of different spectral bands (section 3.1) and explored different combinations for object-based land cover classification (section 3.2). The major steps in the proposed scheme include spatial and spectral feature extractions, image segmentation, and classification. We evaluated the proposed models using reference data and provide comprehensive analyses (section 3.3).

\subsection{Data Combination}

This study analyzed the performance of different spectral data integrated with lidar data. The integration is based on using the same mapping coordinates for lidar and spectral orthoimages, which provide 3D shape information and color information to separate different objects, respectively. The combinations include: (1) lidar integrated with traditional 4-band (blue, green, red, infrared) multispectral images; (2) lidar integrated with advanced 8-band (coastal, blue, green, yellow, red, red edge, nir1, nir2) multispectral images; and (3) lidar integrated with hyper-spectral images. Although the multispectral and hyper-spectral image wavelengths overlap between $400-1050 \mathrm{~nm}$, the band width of a hyper-spectral image is only $10 \mathrm{~nm}$, smaller than that of a multispectral image Therefore, the classification capability (c)

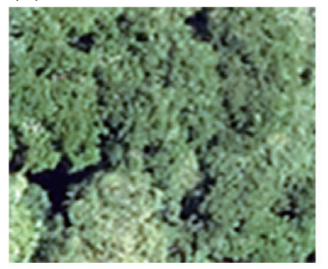

(g)

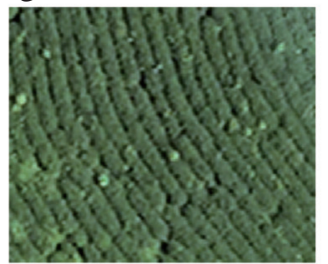

(k)

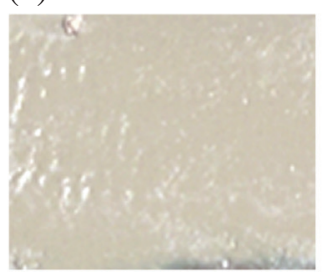

(d)

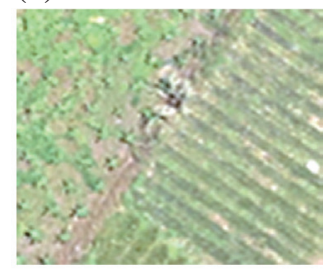

(h)

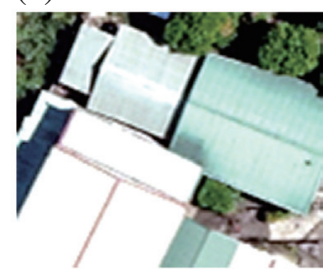

(1)

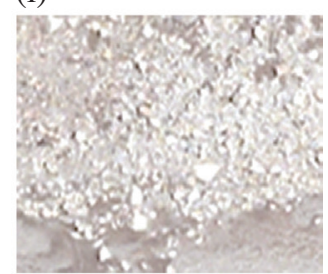

Fig. 2. Targets of classification, (a) areca,(b) bamboo, (c) broadleaf, (d) crop, (e) fruit, (f) grass, (g) tea, (h) building, (i) pavement, (j) bare ground (soil), (k) water, (l) rock. (Color online only) 
(a) Spectrum of traning set (WorldView-2, 8 bands)

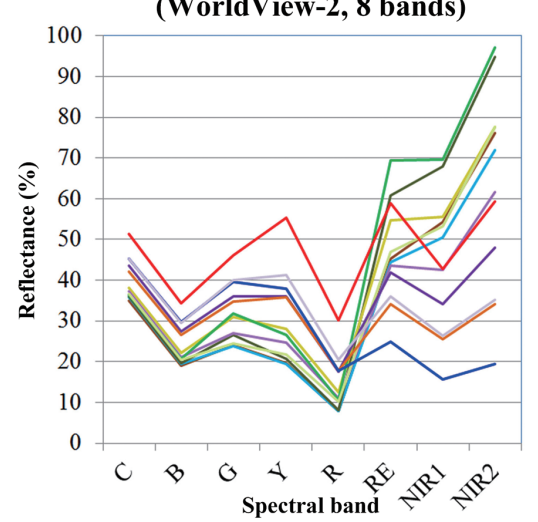

(b) Spectrum of traning set (WorldView-2, 4 bands)

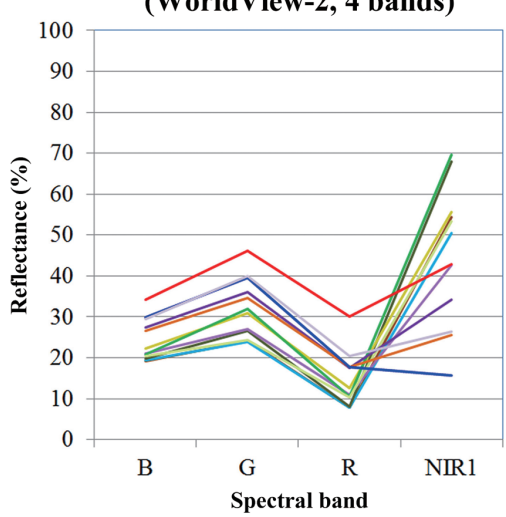

(c) Spectrum of traning set (hyperspectral)

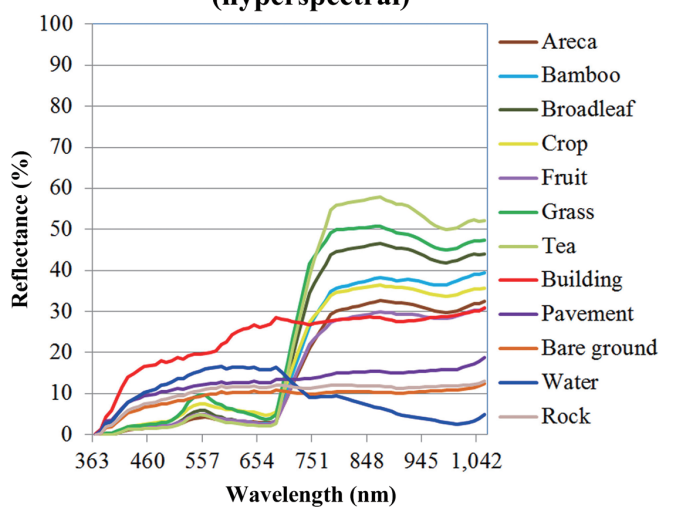

Fig. 3. Comparison of spectral responses for different spectral images (a) 4 bands, (b) 8 bands, (c) hyperspectral image. (Color online only)

(a)

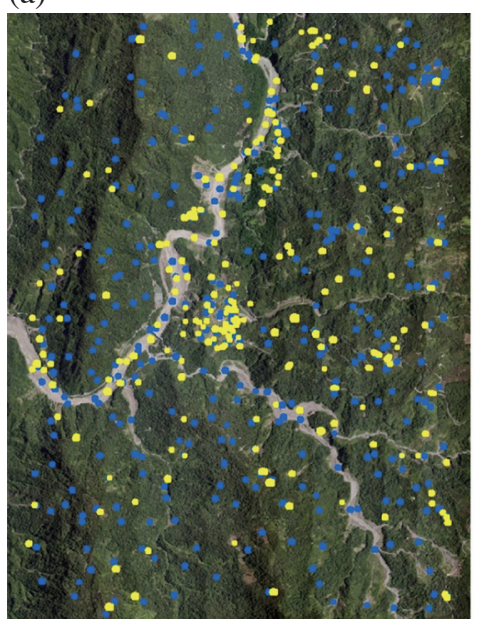

(b)

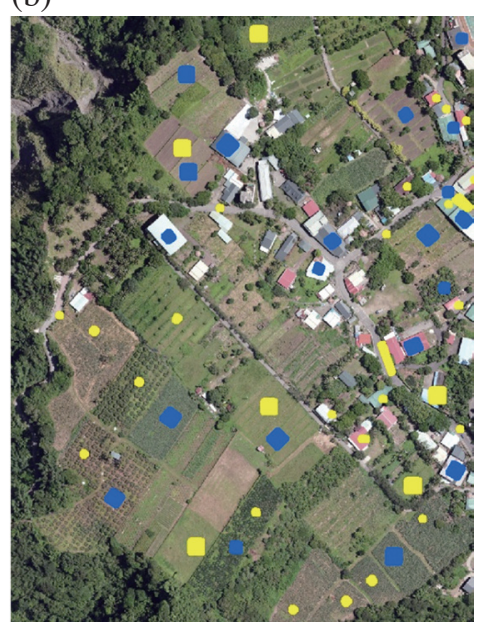

Fig. 4. Distribution of training and check regions (yellow: training area; blue: check area); (a) overview, (b) zoom-in. (Color online only)

Table 2. Summary of training and validation regions.

\begin{tabular}{c|c|c|c|c|c|c|c}
\hline \multicolumn{3}{c|}{ Vegetation } & \multicolumn{5}{c}{ Non-vegetation } \\
\hline & Training $\left(\mathbf{m}^{2}\right)$ & Validation $\left(\mathbf{m}^{2}\right)$ & Total $\left(\mathbf{m}^{2}\right)$ & & Training $\left(\mathbf{m}^{2}\right)$ & Validation $\left(\mathbf{m}^{2}\right)$ & Total $\left(\mathbf{m}^{2}\right)$ \\
\hline Areca & 3000 & 2804 & 5804 & Building & 910 & 1017 & 1927 \\
Bamboo & 3396 & 6737 & 10133 & Pavement & 751 & 1152 & 1903 \\
Broadleaf & 3091 & 6830 & 9921 & Bare ground & 1655 & 1068 & 2723 \\
Crop & 990 & 2241 & 3231 & Water & 1342 & 3808 & 5149 \\
Fruit & 1767 & 998 & 2764 & Rock & 2396 & 1755 & 4151 \\
Grass & 2650 & 1713 & 4363 & & & & \\
Tea & 1894 & 751 & 2645 & & & & \\
\hline Total & 16788 & 22074 & 38862 & Total & 7053 & 8800 & 15854 \\
\hline
\end{tabular}




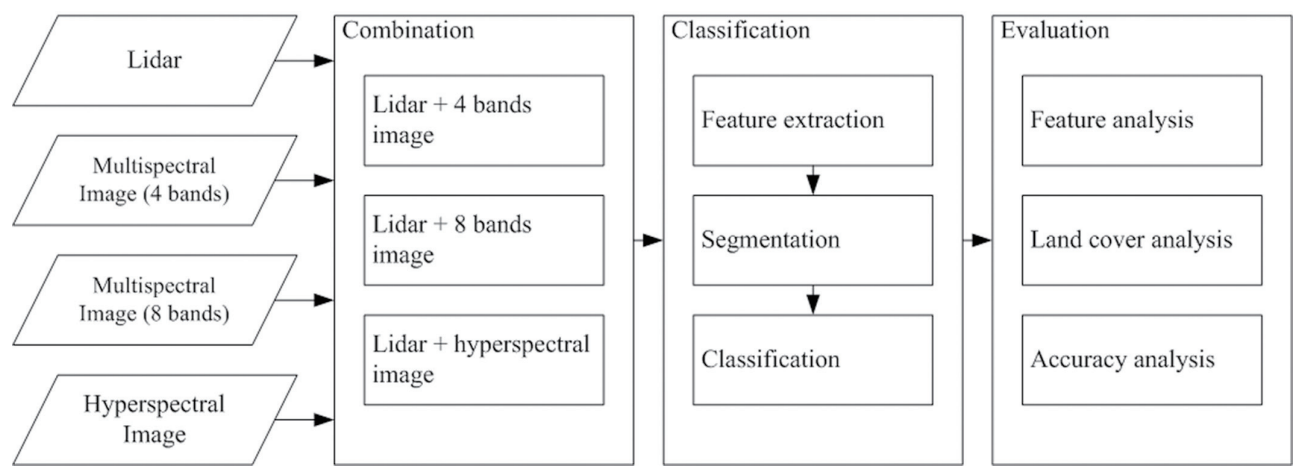

Fig. 5. The proposed scheme.

of hyper-spectral imagery is better than that of multispectral imagery. We quantified the difference between multispectral and hyperspectral images in lidar-assisted classification.

\subsection{Object-Based Classification}

In reality the ground surface objects are composed of a number of pixels. In object-based classification the segmentation aggregates the pixels into an object according to the similarity between pixels. Object-based classification considers the properties of the object (i.e., shape, spatial, and spectral features) and also the relationship between objects, similar to image interpretation from a human perspective. The major components of object-based classification include feature exaction, segmentation and classification.

We calculated different features extracted from different spectral images, lidar and objects (Fig. 6) to identify different land cover types. Lidar features separate different land cover types using a surface feature. The standard deviation in DSM can be treated as surface roughness and the echo ratio parameterizes multiple returns to represent penetration in the vertical direction to identify vegetation and non-vegetation (Höfle et al. 2012). The intensity represents a relative measure for each lidar signal to distinguish pavement from other objects using reflection characteristics. In addition, the DSM textures were applied to separate smooth and rough objects. Spectral features indicate the signature of different land covers from their spectral responses. The segmented object was also used to calculate shape (i.e., area and length-to-width ratio) and texture features (Haralick et al. 1973). The area feature was used to merge small area objects into the nearest object and the length-to-width ratio was used to detect thin objects like rivers and roads.

One advantage of object-based classification is the relationship between objects is applied in post-classification. The use of the area feature is based on the relationship between objects. Theoretically, the same species, likes bamboo, will grow together and cover a certain area. If the area of an object (vegetation type 1) is very small and surrounded by other objects (vegetation type 2), then this object (veg- etation type 1) will be merged into the surrounding objects (vegetation type 2). The concept of this process is similar to the mean filter, which reduces the pepper-and-salt effect in post-classification. All extracted features were summarized (Table 3).

The aim of segmentation is to merge pixels with similar attributes into a region. We used rasterized lidar and spectral orthoimages as the input layers for segmentation and combined elevation attributes from lidar data and radiometric attributes from orthoimages in the segmentation. The segmentation considers both attribute and shape factors. Pixels with similar height and spectral attributes are merged into a region. The attribute is the pixel value of the input layer whereas the shape factor is the shape of the segmented object. The segment concept is based on the heterogeneity index [Eq. (1)] (Baatz and Schäpe 2000). The heterogeneity combines the attribute [Eq. (2)] and shape [Eq. (3)] factors (Baatz and Schäpe 2000). The segmentation is a bottom up method that starts from a pixel. Each pixel is treated as a small object and neighborhood pixels are added to calculate the heterogeneity index. If the heterogeneity index meets the predefined criterion, these pixels are merged together. The advantages of this strategy are: (1) different layers have different weights; (2) the segmentation considers the attribute (pixel value) and also the shape of objects.

$$
\begin{aligned}
& h=w_{\text {attribute }} \times h_{\text {attribute }}+w_{\text {shape }} \times h_{\text {shape }} \\
& h_{\text {attribute }}=\sum_{i=1}^{c} w_{i} \sigma_{i} \\
& h_{\text {shape }}=w_{\text {smooothness }} \times h_{\text {smoothness }}+w_{\text {compactness }} \times h_{\text {compactness }} \\
& h_{\text {smoothness }}=l / b \\
& h_{\text {compactness }}=l / \sqrt{n}
\end{aligned}
$$

where $h$ is heterogeneity index; $h_{\text {attribute }}$ and $h_{\text {shape }}$ are attribute and shape indices; $w_{\text {attribute }}$ and $w_{\text {shape }}$ are weights, $w_{\text {attribute }}+w_{\text {shape }}=1 ; w_{i}$ is weight for layer $i ; \sigma_{i}$ is the standard deviation of layer $i ; h_{\text {smoothness }}$ and $h_{\text {compactness }}$ are smoothness 
(a)

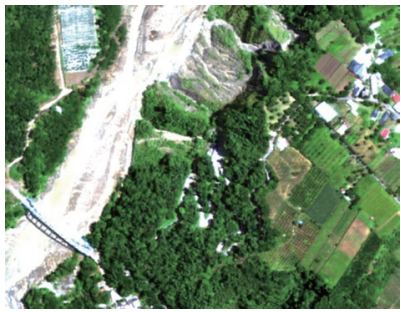

(e)

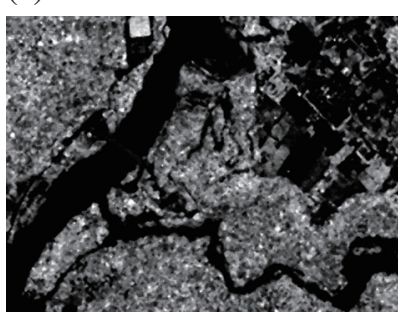

(i)

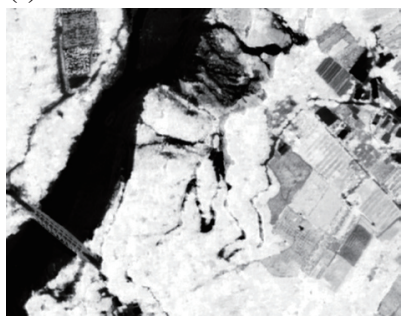

(b)

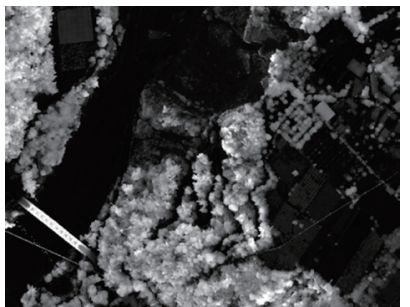

(f)

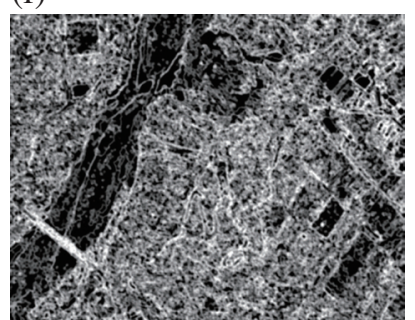

(j)

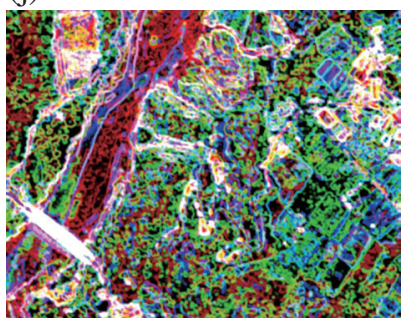

(c)

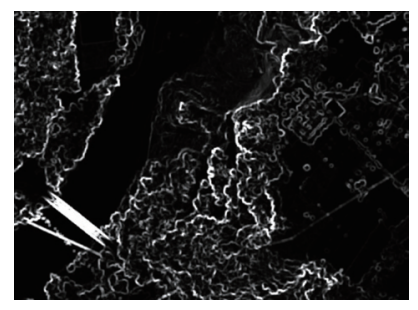

(g)

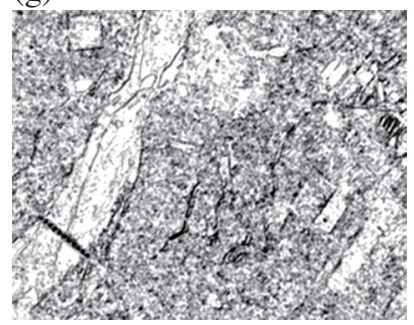

(k)

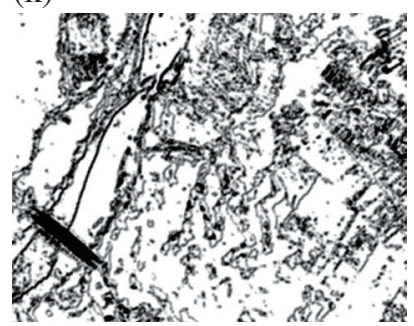

(d)

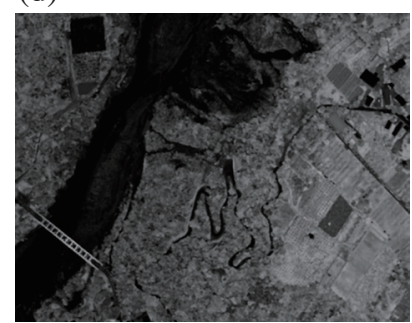

(h)

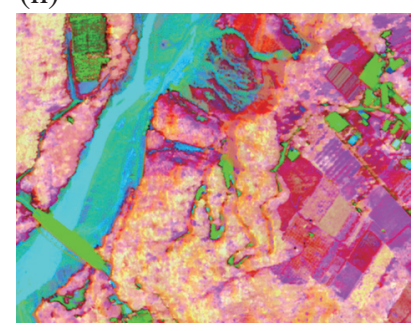

Fig. 6. Illustration of different features (a) optical image, (b) nDSM, (c) roughness, (d) intensity, (e) echo ratio, (f) lidar texture (entropy), (g) lidar texture (homogeneity), (h) MNF, (i) NDVI, (j) image texture (entropy), (k) image texture (homogeneity). (Color online only)

Table 3. Summary of features.

\begin{tabular}{|c|c|c|c|}
\hline Data & Feature type & Feature & Description \\
\hline \multirow{5}{*}{ Lidar } & \multirow{2}{*}{$\begin{array}{l}\text { Terrain } \\
\text { feature }\end{array}$} & $\begin{array}{l}\mathrm{nDSM} \\
\mathrm{nDSM}=\mathrm{DSM}-\mathrm{DTM}\end{array}$ & $\begin{array}{l}\text { nDSM is object height above ground surface. It can be used to distinguish above } \\
\text { ground and ground objects. }\end{array}$ \\
\hline & & $\begin{array}{l}\text { Roughness } \\
\operatorname{STD}(\mathrm{DSM})=\sqrt{\sum(\mathrm{Z}-\overline{\mathrm{Z}})^{2} /(\mathrm{n}-1)}\end{array}$ & $\begin{array}{l}\text { Roughness implies the height complexity in a region. It can be used to distinguish the } \\
\text { smoothness and complexity of an object. }\end{array}$ \\
\hline & \multirow[b]{2}{*}{ Lidar feature } & Intensity & Intensity is the return energy of laser pulse. \\
\hline & & $\begin{array}{l}\text { Echo ratio } \\
\mathrm{ER}=\frac{\mathrm{n}_{\text {first }}+\mathrm{n}_{\text {intermediate }}}{\mathrm{n}_{\text {last }}+\mathrm{n}_{\text {single }}} \times 100 \%\end{array}$ & $\begin{array}{l}\text { A laser pulse may penetrate a vegetation area and produce multiple returns. The Echo } \\
\text { ratio calculates the percentage of first and intermediate returns in a region. A higher } \\
\text { echo ratio has higher possibility for vegetation area. }\end{array}$ \\
\hline & $\begin{array}{l}\text { Texture } \\
\text { feature }\end{array}$ & $\begin{array}{l}\text { GLCM } \\
\text { Entropy }=\sum(P(i, j) \times\{-\ln [P(i, j)]\}) \\
\text { Homogeneity }=\sum\left\{p(i, j) /\left[1+(i-j)^{2}\right]\right\}\end{array}$ & $\begin{array}{l}\text { Lidar texture represents the arrangement and frequency of lidar points in a region. The } \\
\text { entropy and homogeneity indicates the information and smoothness of surface. }\end{array}$ \\
\hline \multirow{3}{*}{$\begin{array}{l}\text { Spectral } \\
\text { image }\end{array}$} & $\begin{array}{l}\text { Spectral } \\
\text { feature }\end{array}$ & MNF & $\begin{array}{l}\text { MNF is used to reduce data redundancy and correlation between bands in hyperspec- } \\
\text { tral images. MNF also transforms original spectral space into new components to } \\
\text { maximize the amount of information (or variance). It can reduce the hyperspectral } \\
\text { image data dimensions. }\end{array}$ \\
\hline & $\begin{array}{l}\text { Spectral } \\
\text { feature }\end{array}$ & $\mathrm{NDVI}=\frac{(\mathrm{NIR}-\mathrm{RED})}{(\mathrm{NIR}+\mathrm{RED})}$ & Normalized vegetation index determine the greenness of land cover. \\
\hline & $\begin{array}{l}\text { Texture } \\
\text { feature }\end{array}$ & $\begin{array}{l}\text { GLCM } \\
\text { Entropy }=\sum(P(i, j) \times\{-\ln [P(i, j)]\}) \\
\text { Homogeneity }=\sum\left\{p(i, j) /\left[1+(i-j)^{2}\right]\right\}\end{array}$ & $\begin{array}{l}\text { Texture of spectral image represents the arrangement and frequency of pixels. The en- } \\
\text { tropy and homogeneity indicates the information and smoothness of spectral response. }\end{array}$ \\
\hline \multirow[b]{2}{*}{ Object } & Shape feature & Area & The size of an object. \\
\hline & Shape feature & Length-to-width ratio $=(\lambda 1) /(\lambda 2)$ & $\begin{array}{l}\text { Length-to-width ratio is used to separate long-and-thin (e.g., road, river) object and } \\
\text { other object (e.g., bare ground). }\end{array}$ \\
\hline
\end{tabular}


and compactness indices, respectively, for shape; $l$ is perimeter; $b$ is smaller length of size; and $n$ is area.

After segmentation an object-based classification rather than pixel-based classification was performed. Each separated region after segmentation was a candidate object for classification. An object-based classification considering the characteristics of elevation, spectral, texture, roughness, and shape information was performed to separate different land cover types. We used a supervised NN classifier in object-based classification. We first manually selected training objects and performed separability analysis to ensure the quality of the training set. The training object attributes were then used in classification. The unknown objects were classified based on the closest training data in the feature space. The NN algorithm uses the mean vectors from the training area to represent each class and searches for the nearest object in the feature space. This algorithm is relatively less complex than that of other algorithms. This study applied many features in the classifications. For this complex combination of object features the NN method is more effective because it requires less setup time for the decision-rule (Laliberte et al. 2006) and was therefore used in this study.

\subsection{Evaluation}

The evaluations were carried out in three steps. We first considered the importance of different features and applied individual features and combined features in classification. The overall accuracy was selected to evaluate the performance of different features and analyze the importance of features. We next compared the classification results using lidar and different spectral images. The performance of different spectral images was qualified in the lidar and spectral image fusion. Third we compared the accuracy of different land covers in different combinations to determine the accuracy of each land cover when different features were available. To discuss the identification of different land covers from different spectral features we used the accuracy index [Eq. (4)] (Pouliot et al. 2002) to evaluate the overall performance for each class. The accuracy index subtracts the omission and commission errors from the correct classification:

$A I=\{[n-(O+C)] / n\} \times 100 \%$

where $n$ is number of correct classifications; $O$ is number of omission errors; and $C$ is the number of commission errors.

\section{RESULTS}

\subsection{Feature Analysis}

In feature analysis we used features from lidar and hyperspectral imagery to analyze the classification perfor- mance for different features. Seven combinations were selected to identify 12 predefined target classes. This study used the commercial software, eCognition ${ }^{\mathrm{TM}} 9$, to perform object-based classification and illustrated the overall and kappa accuracies obtained from different features (Table 4 and Fig. 7). Normalized DSM was selected as the elevation attribute because it is able to separate ground and aboveground objects. We used different radiometric attributes in different combinations. The MNF images were used for hyperspectral images whereas original images were used for multispectral images. We assumed that the importance of elevation and radiometric attributes were the same and therefore used equal weights for all input features. All combinations used the same training area, check areas and segmentation parameters (i.e., scale $=20, h_{\text {attribute }}=0.8$, and $h_{\text {shape }}=0.2$ ). The shape features can be obtained directly from the segmented objects; therefore, we applied this shape feature in all combinations (Table 4). Combinations 1 to 3 used lidar features only. The more lidar features were involved the higher the overall accuracy, but the overall accuracy reached only $57.92 \%$ when all of the lidar features were adopted due to the number of pre-defined target classes. Lidar features were usually used to classify roads, trees, grass, bare ground, and buildings (Guo et al. 2011; Zhou 2013; Wang et al. 2014). The classification accuracy for these 5 land covers reached $80 \%$ because they have significant differences in shape and intensity. For example, the intensity of an asphalt road is significantly lower than that for other objects, and the penetration rate for trees is significantly higher than that for other objects. The nDSM is a useful feature to effectively separate bare ground from above-ground objects. We defined 12 target classes in this study for classification, several of which could not be separated using lidar features only. For example, crops and grass have similar spatial features and therefore additional spectral features were needed to identify different land covers in complex areas.

Combinations 4 to 6 represent the classification results using spectral features. The spectrally transformed MNF (combination 4) was able to provide $75.40 \%$ accuracy, better than the lidar features results. The NDVI is a useful index for separating vegetation from non-vegetation. Combination 5 combined MNF and NDVI in classification, but the improvement was not significant because the hyperspectral MNF is able to separate vegetation from non-vegetation (Fig. 2c). Combination 6 added texture features to improve the classification accuracy. The smoothness and roughness of spectral responses are spectrally derived features that separate smooth and rough objects.

Combination 7 was the result from using both lidar and spectral features, and performed better than the other combinations. Comparing the results from all features and spectral only, the improvement was about 6\% (i.e., 76.66 - 83.08\%), but the improvement rate for all features and lidar only was about 25\% (i.e., 57.92 - 83.08\%). Zhou (2013) also found 
that the combination of lidar and spectral imaging improved the land cover classification accuracy. The spectral features were more suitable for separating different species (e.g., crops vs. grass) whereas lidar features were more suitable for distinguishing objects with different shapes (e.g., building vs. tree). This analysis clearly showed the advantages of feature fusion for land cover classification.

This study analyzed the classification results from lidar and different spectral images. The analysis of individual lidar features are shown as combinations 1 to 3 and the hyperspectral features analyses are shown as combinations 4 to 6 . For lidar features the echo ratio improves the identification of tree and non-tree, and the roughness and texture improve the classification of smooth (e.g., road) and irregular objects (e.g., rock). For the hyperspectral features, the spectral features from MNF transformation are the most important features but NDVI and texture features are limited. We analyzed the feature contributions using error matrices from these 6 combinations and found that: (1) the echo ratio from lidar improves the accuracies for crops, fruit, bare ground, and buildings because these four classes have signification differences in echo ratio; (2) the lidar texture improves the accuracies for pavement, bare ground, tea, and grass because these four classes have signification differences in shape; and (3) the spectral texture improves the accuracies for areca and fruit cover.

The benefit of lidar texture is in identifying objects with smooth and rough surfaces. For example, the road (pavement) surface is usually smoother than rock and bare ground. The improvement with additional texture features reaches $6 \%$ overall accuracy. The accuracy of hyperspectral imaging is contributed mainly by MNF images, but the improvement from spectral texture is limited in this study area. The shape features (i.e., area and length-to-width) were applied in post-processing. The area of objects was used to reduce the number of small objects (i.e., area $<50 \mathrm{~m}^{2}$ ). We merged the smaller objects and connected them to other objects. The length-to-width ratio was used to separate long, thin (e.g., road) objects from other objects (e.g., bare ground).

The segmentation parameters included scale, weight of shape and weight of color. Among these parameters the scale parameter is more sensitive than others. The scale

Table 4. Features analysis for combinations 1 to 7.

\begin{tabular}{|c|c|c|c|c|c|c|c|}
\hline \multirow{2}{*}{ Combinations } & \multirow{2}{*}{ Input Features } & \multicolumn{4}{|c|}{ Features for segmentation } & \multirow{2}{*}{ OA $(\%)$} & \multirow{2}{*}{ Kappa } \\
\hline & & Feature & Weight & Feature & Weight & & \\
\hline 1 & Lidar: nDSM, Intensity & $\mathrm{nDSM}$ & 0.5 & Intensity & 0.5 & 45.08 & 0.363 \\
\hline 2 & Lidar: nDSM, Intensity, Echo ratio & $\mathrm{nDSM}$ & 0.5 & Intensity & 0.5 & 51.19 & 0.425 \\
\hline 3 & Lidar: nDSM, Intensity, Echo ratio, Roughness, Texture & $\mathrm{nDSM}$ & 0.5 & Intensity & 0.5 & 57.92 & 0.507 \\
\hline 4 & Hyperspectral: MNF & & & 7 MNF bands & $1 / 7$ & 75.40 & 0.714 \\
\hline 5 & Hyperspectral: MNF, NDVI & & & 7 MNF bands & $1 / 7$ & 75.60 & 0.715 \\
\hline 6 & Hyperspectral: MNF, NDVI, Texture & & & 7 MNF bands & $1 / 7$ & 76.66 & 0.783 \\
\hline 7 & $\begin{array}{l}\text { All } 8 \text { features: nDSM, Intensity, Echo ratio, Roughness, } \\
\text { Texture (Lidar), MNF, NDVI, Texture (MNF) }\end{array}$ & $\mathrm{nDSM}$ & 0.5 & $7 \mathrm{MNF}$ bands & $0.5 *(1 / 7)$ & 83.08 & 0.802 \\
\hline
\end{tabular}

\section{Accuracies of Different Combinations}

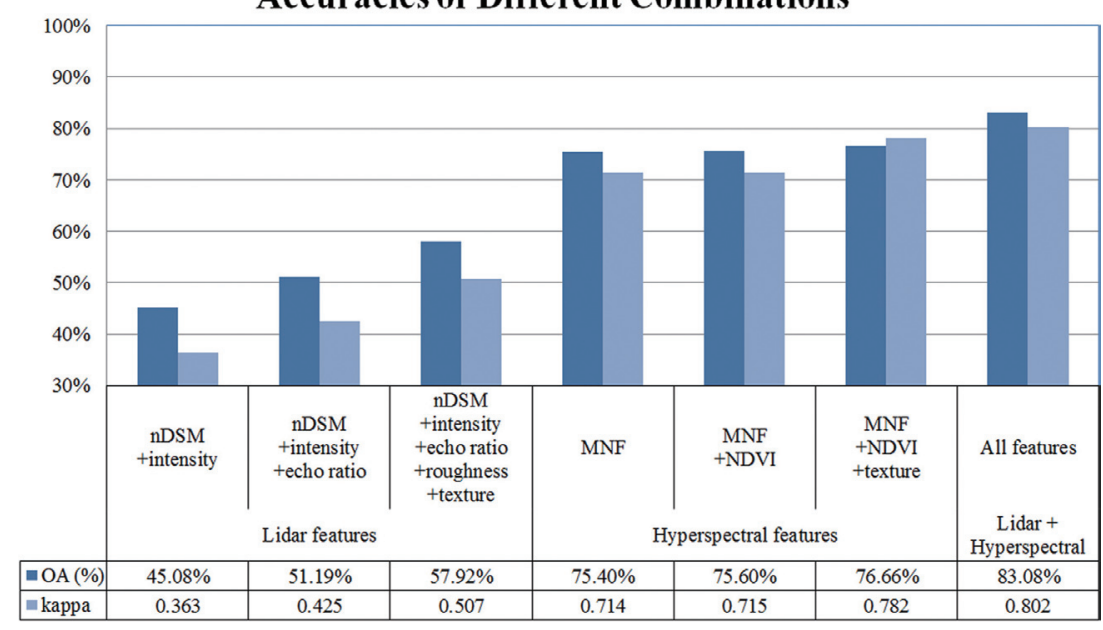

Fig. 7. Comparison of different combinations. (Color online only) 
parameter selection depends on the scale of the phenomenon of interest (Kim and Madden 2006; Addink et al. 2007). We selected several scales (i.e., 10, 20, 30, and 40) for combination 7 in segmentation and classification to understand the sensitivity of the segment parameters (Fig. 8). The smaller scale (i.e., 10) may cause over-segmentation, resulting in an overall accuracy less than $80 \%$. By comparison, the larger scale parameter (i.e., 40) may cause under-segmentation, also resulting in overall accuracy less than $80 \%$. Higher accuracy occurs at scale 20 . Therefore, this study adopted this parameter for all combinations in accuracy analysis.

\subsection{Comparison of Different Spectral Images}

We compared the lidar results with different spectral images (Table 5 and Fig. 9). The selected features, training areas, check areas and classification method were the same except for spectral images. The input spectral images included hyperspectral images and 4- and 8-band multispectral images. Four-band multispectral imagery is the most common spectral image used. The improvement in results comparing 4-band (combination 9) to 8-band (combination 8) multispectral images was about $9.05 \%$ (i.e., $60.98-70.03 \%$ ), indicating that the advanced 8-band multispectral images enhanced the land cover mapping by including additional coastal, yellow, red edge, and nir2 bands. The spectral responses (Fig. 2) can be used to explain the improvement in 8-band imagery.
The seperability of different land covers for 8-band images (Fig. 2b) is better than that from 4-band images (Fig. 2a), demonstrating the superior accuracy of 8-band images, further demonstrated by (Elsharkawy et al. 2012) for 7 types of land covers. They used only multispectral images for 7 classes and showed an improvement rate of about $2 \%$. Because we defined 12 classes in classification the improvement rate was higher than that from the previous study; hence, the advanced 8-band multispectral imagery is more suitable for detailed classification.

The 8-band multispectral image and hyperspectral image wavelengths are similar and overlapped between $400-1050 \mathrm{~nm}$, but the bandwidth of hyperspectral (i.e., $10 \mathrm{~nm}$ ) is narrower than the bandwidth of multispectral imagery $(>100 \mathrm{~nm})$. Because hyperspectral imagery has more detailed spectral information the improvement in hyperspectral and 8-band was about $13.05 \%$ (i.e., 83.08 - 70.03\%). The improvement rate with versus without lidar was about $6 \%$ (section 4.1), clearly indicating that the spectral information significantly contributes to land cover classification.

\subsection{Land Cover Analysis}

To understand the effect of spectral features for different land cover types we used completeness (also called producer accuracy, Fig. 10a), correctness (also called user accuracy, Fig. 10b), and an accuracy index (Table 6) for

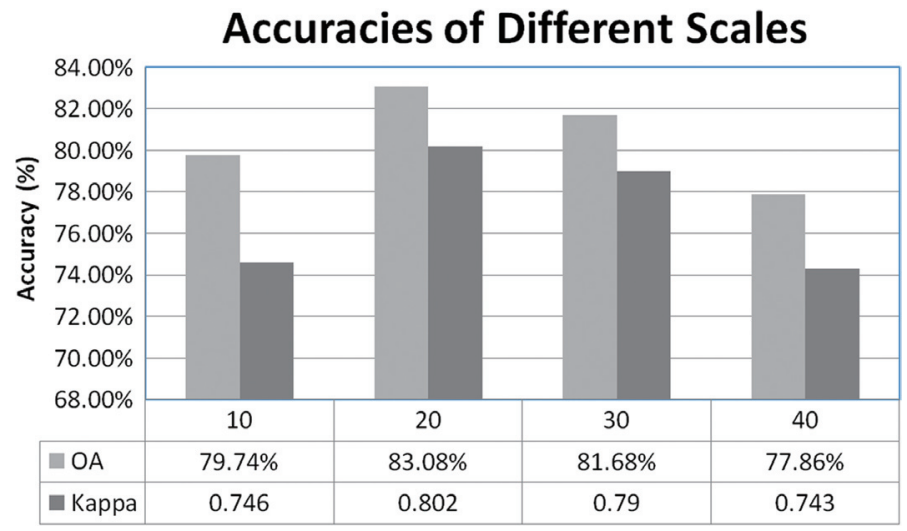

Fig. 8 . The analysis of scale parameters. (Color online only)

Table 5. Features analysis for combinations 7 to 9 .

\begin{tabular}{|c|c|c|c|c|c|c|c|}
\hline \multirow{2}{*}{ Combinations } & \multirow{2}{*}{ Input Features } & \multicolumn{4}{|c|}{ Features for segmentation } & \multirow{2}{*}{ OA $(\%)$} & \multirow{2}{*}{ Kappa } \\
\hline & & Feature & Weight & Feature & Weight & & \\
\hline 7 & $\begin{array}{l}\text { Lidar + hyperspectral image: nDSM, Intensity, Echo ratio, } \\
\text { Roughness, Texture (Lidar), MNF, NDVI, Texture (MNF) }\end{array}$ & $\mathrm{nDSM}$ & 0.5 & $7 \mathrm{MNF}$ bands & $0.5 *(1 / 7)$ & 83.08 & 0.802 \\
\hline 8 & $\begin{array}{c}\text { Lidar }+8 \text { bands multispectral image: nDSM, Intensity, Echo ratio, } \\
\text { Roughness, Texture (Lidar), } 8 \text { bands, NDVI, Texture (image) }\end{array}$ & $\mathrm{nDSM}$ & 0.5 & 8 bands & $0.5^{*}(1 / 8)$ & 70.03 & 0.652 \\
\hline 9 & $\begin{array}{c}\text { Lidar }+4 \text { bands multispectral image: nDSM, Intensity, Echo ratio, } \\
\text { Roughness, Texture (Lidar), } 4 \text { bands, NDVI, Texture (image) }\end{array}$ & $\mathrm{nDSM}$ & 0.5 & 4 bands & $0.5^{*}(1 / 4)$ & 60.98 & 0.550 \\
\hline
\end{tabular}


(a)

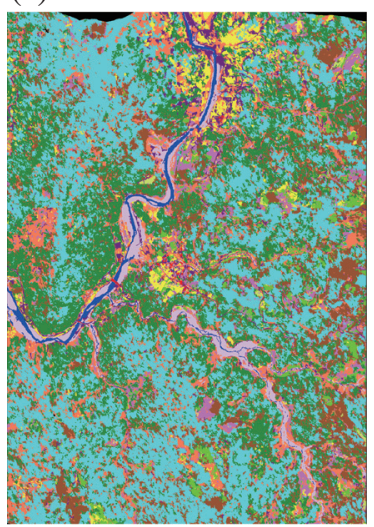

(c)

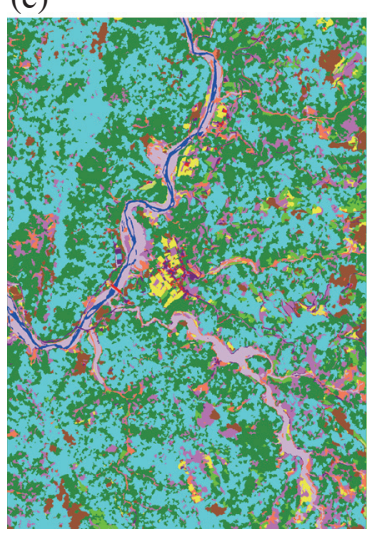

(b)

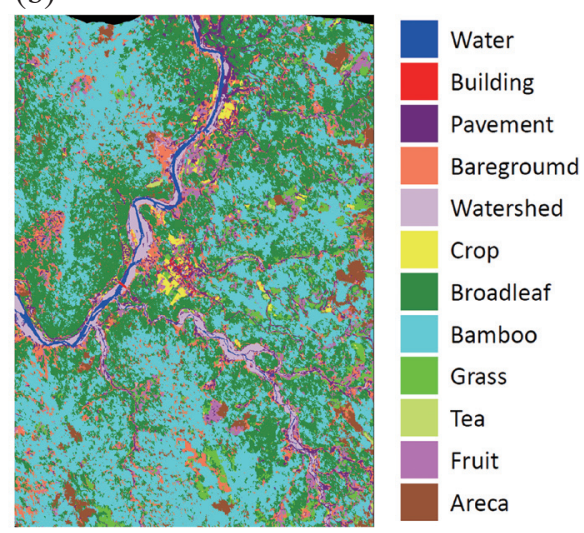

(d)

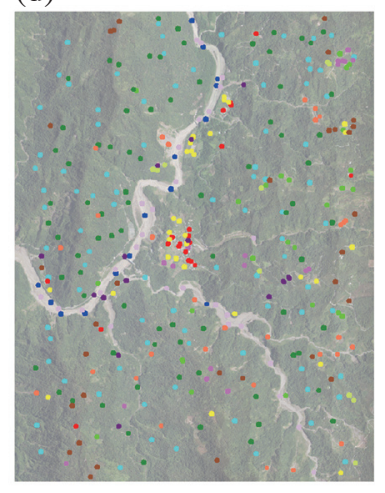

Fig. 9. Classification results for different combinations (a) lidar and 4 bands image, (b) lidar and 8 bands image, (c) lidar and hyperspectral image, (d) distribution of check areas. (Color online only)
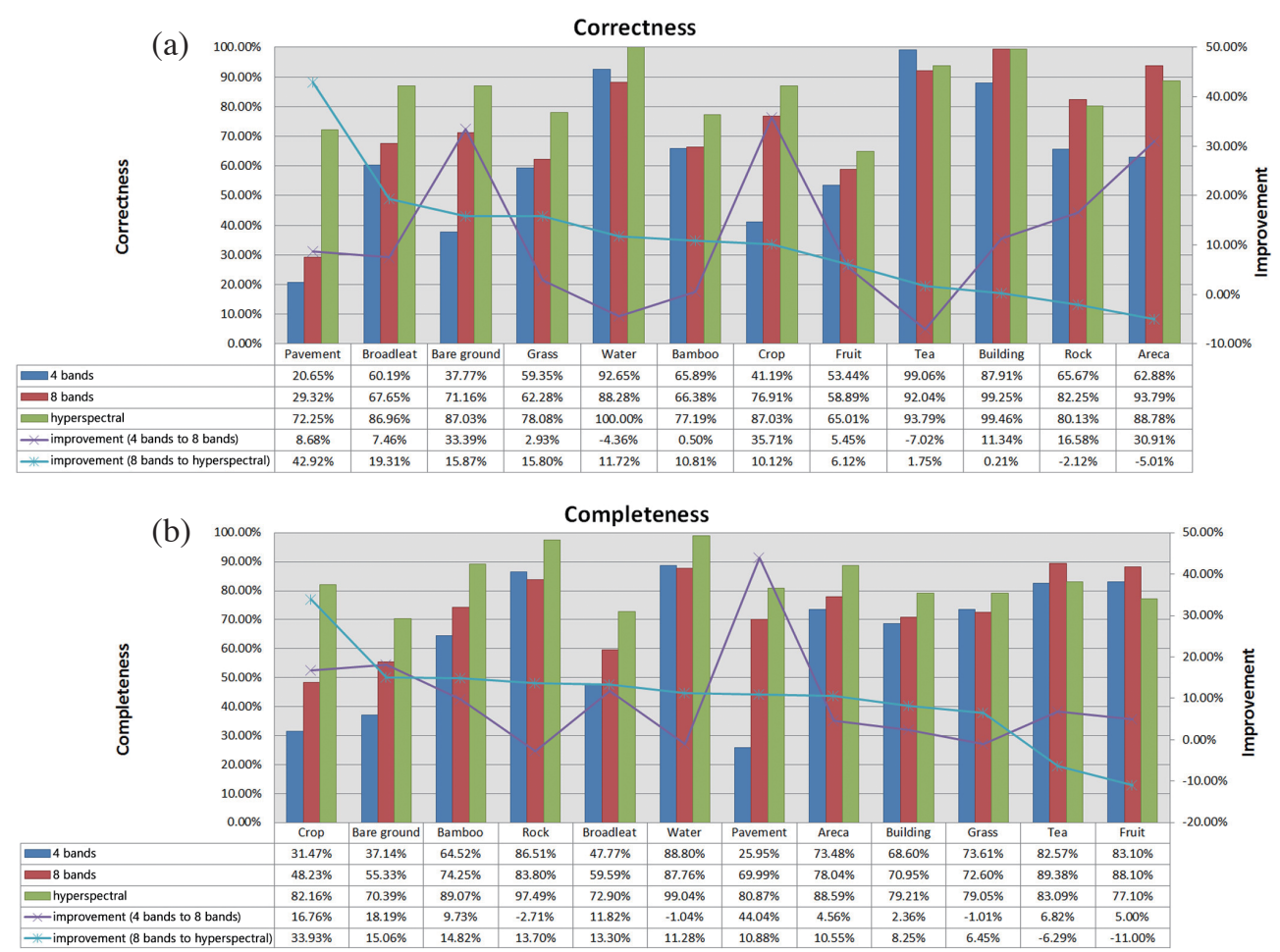

Fig. 10. Correctness and completeness for different classes, (a) correctness (b) completeness. (Color online only) 
Table 6. Accuracy indices for combinations 7 to 9 .

\begin{tabular}{cccccc}
\hline & $\begin{array}{c}\text { Combination 9 } \\
\text { Lidar + 4 Bands }\end{array}$ & $\begin{array}{c}\text { Combination 8 } \\
\text { Lidar + 8 Bands }\end{array}$ & $\begin{array}{c}\text { Combination 7 } \\
\text { Lidar + Hyper }\end{array}$ & $\begin{array}{c}\text { Improvement (4 } \\
\text { to 8 bands) }\end{array}$ & $\begin{array}{c}\text { Improvement (8 } \\
\text { bands to Hyper) }\end{array}$ \\
\hline Areca & $31.4 \%$ & $76.0 \%$ & $77.4 \%$ & $\mathbf{4 4 . 6 \%}$ & $1.4 \%$ \\
Bamboo & $31.1 \%$ & $36.6 \%$ & $62.8 \%$ & $5.5 \%$ & $26.1 \%$ \\
Broadleaf & $16.2 \%$ & $31.1 \%$ & $62.0 \%$ & $14.9 \%$ & $\mathbf{3 0 . 9 \%}$ \\
Crop & $-13.5 \%$ & $33.8 \%$ & $69.9 \%$ & $\mathbf{4 7 . 2 \%}$ & $\mathbf{3 6 . 2 \%}$ \\
Fruit & $10.7 \%$ & $26.6 \%$ & $35.6 \%$ & $15.9 \%$ & $9.0 \%$ \\
Grass & $23.2 \%$ & $28.6 \%$ & $56.9 \%$ & $5.4 \%$ & $28.2 \%$ \\
Tea & $81.8 \%$ & $81.7 \%$ & $77.6 \%$ & $-0.1 \%$ & $-4.1 \%$ \\
Bare ground & $-24.0 \%$ & $32.9 \%$ & $59.9 \%$ & $57.0 \%$ & $27.0 \%$ \\
Building & $59.2 \%$ & $70.4 \%$ & $78.8 \%$ & $11.3 \%$ & $8.4 \%$ \\
Pavement & $-73.8 \%$ & $-98.7 \%$ & $49.8 \%$ & $-24.9 \%$ & $\mathbf{1 4 8 . 5 \%}$ \\
Rock & $41.3 \%$ & $65.7 \%$ & $73.3 \%$ & $24.4 \%$ & $7.6 \%$ \\
Water & $81.8 \%$ & $76.1 \%$ & $99.0 \%$ & $-5.6 \%$ & $22.9 \%$ \\
\hline
\end{tabular}

each land cover in different combinations. Correctness measures the percentage of extracted classes correctly classified, whereas completeness measures the percentage of classes in the reference data. Statistical analysis indicated that the results from lidar and hyperspectral images are significantly improved compared to the results from those produced from lidar and multispectral images. The results indicate the benefit of using narrow spectral rather than broad spectral bands in species classification. The use of additional lidar spatial features has improved the separability of above ground and on ground objects.

The correctness analyses for crops, bare ground and pavement for 4-band multispectral images was less than $50 \%$ because old pavement was similar to the behavior of bare ground, and dry crops with low chlorophyll was similar to bare ground. With the additional spectral bands the 8-band multispectral imagery improved the accuracy of these three classes from $8.68-35.71 \%$. The use of yellow and red edge bands provided better discrimination between dry crops and bare ground. Overall, the correctness improvement from 8-band multispectral images to hyperspectral images ranged from $0.21-42.92 \%$. For vegetation classes the improvement in broadleaf, bamboo, grass, and crops were better than $10 \%$, but the improvement in areca was $-5.01 \%$ when compared to 8-band images because areca was slightly misclassified as bamboo.

In completeness analysis, the behavior of crop, bare ground, and pavement was similar to correctness. With the additional spectral bands, the 8-band multispectral imagery improved the accuracy of these three classes from $16.76-44.04 \%$. Overall, the improvement of completeness for 8-band multispectral and hyperspectral image ranged from $6.45-33.93 \%$. Because the tea and fruit covers were misclassified as grass, the improvement of tea and fruit were -6.29 and $-11.00 \%$. With the exception of tea and fruit, the improvement rates for different land covers were higher than $5 \%$.

This study also used an accuracy index to evaluate the overall performance for each class (Table 6). Comparing the accuracy indices between 4- and 8-band multispectral images, bare ground, crops, and areca had accuracy values higher than $40 \%$. The additional bands (i.e., coastal, yellow, red edge, and nir2) significantly improved the discrimination for these three classes. With the hyperspectral image the accuracies for pavement, crops and broadleaf were improved better than $30 \%$. Moreover, bamboo, grass, bare ground, and water showed moderate improvements when hyperspectral images were available. Most accuracy indices for hyperspectral images were higher than those for multispectral images.

\section{CONCLUSIONS}

This study proposed an object-based land cover classification method using a combination of lidar and different spectral images. The hyperspectral images, 4- and 8-band multispectral WorldView-2 satellite images were compared. The major contributions of this study are to (1) establish an object-based land cover classification scheme using lidar and spectral features; (2) compare classification lidar results with those from different spectral images; and (3) evaluate the improvement rate for different land covers when different features are available. The proposed scheme integrated spatial lidar features and spectral features to identify different land cover types, subsequently improving the accuracy of land cover mapping.

We conclude the following: (1) The integration of hyperspectral images and lidar has higher accuracy than hyperspectral only and lidar only. The improvement rate reached $6 \%$ for the data fusion approach. The combination 
of spatial and spectral data is beneficial for land cover identification. (2) For the comparison of traditional 4-band and advanced 8-band multispectral images in data fusion the improvement rate of 8-band images reached $9 \%$ for 12 classes. The additional coastal, yellow, red edge, and nir 2 are useful for land cover mapping. The additional bands lead to improvement in the classification accuracies for areca, crop, and bare ground. (3) Lidar features are useful for separating man-made objects and vegetation, whereas spectral features are useful for separating different vegetation types. In the 8-band multispectral images comparison with hyperspectral images, using narrow spectral (hyperspectral) bands has better accuracy than broad spectral bands (WorldView-2) in species classification. The improvement rate of hyperspectral images reached $13 \%$ for 12 classes. Future works will focus on examining different classifiers (e.g., SVM) for object-based classification.

Acknowledgements The authors would like to thank the Ministry of Interior of Taiwan (project number SYC1030219) for the providing test data and funding support.

\section{REFERENCES}

Addink, E. A., S. M. de Jong, and E. J. Pebesma, 2007: The importance of scale in object-based mapping of vegetation parameters with hyperspectral imagery. Photogramm. Eng. Rem. Sens., 73, 905-912, doi: 10.14358/ PERS.73.8.905. [Link]

Baatz, M. and A. Schäpe, 2000: Multiresolution segmentation: an optimization approach for high quality multiscale image segmentation. In: Strobl, J., T. Blaschke, and G. Griesebner (Eds.), Angewandte Geographische Informations Verarbeitung XII, Wichmann-Verlag, Heidelberg, 12-23. Available at http://www.ecognition.com/sites/default/files/405_baatz_fp_12.pdf.

Bioucas-Dias, J. M., A. Plaza, G. Camps-Valls, P. Scheunders, N. M. Nasrabadi, and J. Chanussot, 2013: Hyperspectral remote sensing data analysis and future challenges. IEEE Geosci. Rem. Sens. Mag., 1, 6-36, doi: 10.1109/MGRS.2013.2244672. [Link]

Blaschke, T., 2010: Object based image analysis for remote sensing. ISPRS J. Photogram. Rem. Sens., 65, 2-16, doi: 10.1016/j.isprsjprs.2009.06.004. [Link]

Bork, E. W. and J. G. Su, 2007: Integrating LIDAR data and multispectral imagery for enhanced classification of rangeland vegetation: A meta analysis. Remote Sens. Environ., 111, 11-24, doi: 10.1016/j.rse.2007.03.011. [Link]

Chen, L. C., T. A. Teo, and J. Y. Wen, and J. Y. Rau, 2007: Occlusion-compensated true orthorectification for high-resolution satellite images. Photogramm. Rec., 22, 39-52, doi: 10.1111/j.1477-9730.2007.00416.x. [Link]
Chen, L. C., C. Y. Huang, and T. A. Teo, 2012: Multi-type change detection of building models by integrating spatial and spectral information. Int. J. Remote Sens., 33, 1655-1681, doi: 10.1080/01431161.2011.593584. [Link]

Chen, Y., W. Su, J. Li, and Z. Sun, 2009: Hierarchical object oriented classification using very high resolution imagery and LIDAR data over urban areas. Adv. Space Res., 43, 1101-1110, doi: 10.1016/j.asr.2008.11.008. [Link]

Clark, M. L., D. A. Roberts, and D. B. Clark, 2005: Hyperspectral discrimination of tropical rain forest tree species at leaf to crown scales. Remote Sens. Environ., 96, 375-398, doi: 10.1016/j.rse.2005.03.009. [Link]

Dalponte, M., L. Bruzzone, and D. Gianelle, 2008: Fusion of hyperspectral and LIDAR remote sensing data for classification of complex forest areas. IEEE Trans. Geosci. Remote Sensing, 46, 1416-1427, doi: 10.1109/ TGRS.2008.916480. [Link]

Debes, C., A. Merentitis, R. Heremans, J. Hahn, N. Frangiadakis, T. van Kasteren, W. Liao, R. Bellens, A. Pižurica, S. Gautama, W. Philips, S. Prasad, Q. Du, and F. Pacifici, 2014: Hyperspectral and LiDAR data fusion: Outcome of the 2013 GRSS data fusion contest. IEEE J. Sel. Top. Appl. Earth Obs. Rem. Sens., 7, 2405-2418, doi: 10.1109/JSTARS.2014.2305441. [Link]

Duro, D. C., S. E. Franklin, and M. G. Dubé, 2012: Multiscale object-based image analysis and feature selection of multi-sensor earth observation imagery using random forests. Int. J. Remote Sens., 33, 4502-4526, doi: 10.1080/01431161.2011.649864. [Link]

Elsharkawy, A., M. Elhabiby, and N. El-Sheimy, 2012: Improvement in the detection of land cover classes using the Worldview-2 imagery. ASPRS 2012 Annual Conference, Sacramento, California, March 19-23, 2012, $11 \mathrm{pp}$.

Gamba, P., 2014: Image and data fusion in remote sensing of urban areas: status issues and research trends. Int. J. Image Data Fusion, 5, 2-12, doi: 10.1080/19479832.2013.848477. [Link]

Gao, Y. and J. F. Mas, 2008: A comparison of the performance of pixel-based and object-based classifications over images with various spatial resolutions. GEOBIA 2008 - Pixels, Objects, Intelligence, GEOgraphic Object Based Image Analysis for the $21^{\text {st }}$ Century, Vol. XXXVIII-4/C1, 6 pp. Available at http:// www.isprs.org/proceedings/XXXVIII/4-C1/Sessions/ Session1/6589_Y_Gao_Proc_pap.pdf.

Geerling, G. W., M. Labrador-Garcia, J. G. P. W. Clevers, A. M. J. Ragas, and A. J. M. Smits, 2007: Classification of floodplain vegetation by data fusion of spectral (CASI) and LiDAR data. Int. J. Remote Sens., 28, 4263-4284, doi: 10.1080/01431160701241720. [Link] 
Govender, M., K. Chetty, V. Naiken, and H. Bulcock, 2008: A comparison of satellite hyperspectral and multispectral remote sensing imagery for improved classification and mapping of vegetation. Water SA, 34, 147-154.

Guo, L., N. Chehata, C. Mallet, and S. Boukir, 2011: Relevance of airborne lidar and multispectral image data for urban scene classification using Random Forests. ISPRS J. Photogram. Rem. Sens., 66, 56-66, doi: 10.1016/j.isprsjprs.2010.08.007. [Link]

Haralick, R. M., K. Shanmugam, and I. Dinstein, 1973: Textural features for image classification. IEEE Trans. Syst. Man. Cybern., SMC-3, 610-621, doi: 10.1109/ TSMC.1973.4309314. [Link]

Höfle, B., M. Hollaus, and J. Hagenauer, 2012: Urban vegetation detection using radiometrically calibrated smallfootprint full-waveform airborne LiDAR data. ISPRS J. Photogram. Rem. Sens., 67, 134-147, doi: 10.1016/j. isprsjprs.2011.12.003. [Link]

Hong, T. M., K. J. Wu, and C. K. Wang, 2013: The effect of topographic factor in atmospheric correction for hyperspectral data. Proceeding of the 34th Asian Conference on Remote Sensing, CD-Rom. Available at http://a-a-rs.org/acrs/index.php/acrs/acrs-overview/proceedings1 ? view=publication \& task=show \&id $=1175$.

Immitzer, M., C. Atzberger, and T. Koukal, 2012: Tree species classification with random forest using very high spatial resolution 8-band Worldview-2 satellite data. Remote Sens., 4, 2661-2693, doi: 10.3390/rs4092661. [Link]

Ke, Y., L. J. Quackenbush, and J. Im, 2010: Synergistic use of QuickBird multispectral imagery and LIDAR data for object-based forest species classification. $R e$ mote Sens. Environ., 114, 1141-1154, doi: 10.1016/j. rse.2010.01.002. [Link]

Kim, M. and M. Madden, 2006: Determination of optimal scale parameter for alliance-level forest classification of multispectral Ikonos images. Bridging Remote Sensing and GIS, 1st International Conference on Objectbased Image Analysis (OBIA 2006), Vol. XXXVI-4/ C42, 4 pp. Available at http://www.isprs.org/proceedings/XXXVI/4-C42/Papers/03_Automated\%20classification\%20Forest/OBIA2006_Kim_Madden.pdf.

Laliberte, A. S., J. Koppa, E. L. Fredrickson, and A. Rango, 2006: Comparison of nearest neighbor and rule-based decision tree classification in an object-oriented environment. 2006 IEEE International Symposium on Geoscience and Remote Sensing, Denver, CO, USA, 3923-3926, doi: 10.1109/IGARSS.2006.1006. [Link]

Liao, W., R. Bellens, A. Pižurica, S. Gautama, and W. Philips, 2014: Combining feature fusion and decision fusion for classification of hyperspectral and LiDAR data. 2014 IEEE Geoscience and Remote Sensing Symposium, Quebec City, QC, 1241-1244, doi: 10.1109/ IGARSS .2014.6946657. [Link]

Liao, W., X. Huang, F. Van Coillie, S. Gautama, A. Pižurica, W. Philips, H. Liu, T. Zhu, M. Shimoni, G. Moser, and D. Tuia, 2015: Processing of multiresolution thermal hyperspectral and digital color data: Outcome of the 2014 IEEE GRSS data fusion contest. IEEE J. Sel. Top. Appl. Earth Obs. Rem. Sens., 8, 2984-2996, doi: 10.1109/JSTARS.2015.2420582. [Link]

Longbotham, N., C. Chaapel, L. Bleiler, C. Padwick, W. J. Emery, and F. Pacifici, 2012: Very high resolution multiangle urban classification analysis. IEEE Trans. Geosci.Remote Sensing, 50, 1155-1170, doi: 10.1109/ TGRS.2011.2165548. [Link]

Myint, S. W., P. Gober, A. Brazel, S. Grossman-Clarke, and Q. Weng, 2011: Per-pixel vs. object-based classification of urban land cover extraction using high spatial resolution imagery. Remote Sens. Environ., 115, 11451161, doi: 10.1016/j.rse.2010.12.017. [Link]

Pohl, C. and J. L. Van Genderen, 1998: Multisensor image fusion in remote sensing: Concepts, methods and applications. Int. J. Remote Sens., 19, 823-854, doi: 10.1080/014311698215748. [Link]

Pouliot, D. A., D. J. King, F. W. Bell, and D. G. Pitt, 2002: Automated tree crown detection and delineation in high-resolution digital camera imagery of coniferous forest regeneration. Remote Sens. Environ., 82, 322334, doi: 10.1016/S0034-4257(02)00050-0. [Link]

Rahman, M. Z. A., W. H. W. Kadir, A. W. Rasib, A. Ariffin, and K. A. Razak, 2013: Integration of high density airborne LiDAR and high spatial resolution image for landcover classification. 2013 IEEE International Geoscience and Remote Sensing Symposium - IGARSS, Melbourne, VIC, 2927-2930, doi: 10.1109/IGARSS.2013.6723438. [Link]

Updike, T. and C. Comp, 2010: Radiometric use of WorldView-2 imagery. DigitalGlobe Technical Note, Revision $1.0,17 \mathrm{pp}$. Available at http://global.digitalglobe.

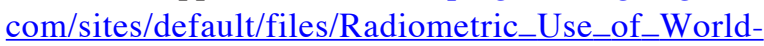
View-2_Imagery\%20(1).pdf.

Wang, C. K., Y. H. Tseng, and H. J. Chu, 2014: Airborne dual-wavelength LiDAR data for classifying land cover. Remote Sens., 6, 700-715, doi: 10.3390/rs6010700. [Link]

Zhou, W., 2013: An object-based approach for urban land cover classification: integrating LiDAR height and intensity data. IEEE Geosci. Rem. Sens. Lett., 10, 928931, doi: 10.1109/LGRS.2013.2251453. [Link] 